\title{
Sinopsis de la Situación de Salud Oral en Chile - Parte I: Garantías Explícitas y Guías Clínicas.
}

\section{Synopsis of the Oral Health Situation in Chile - Part I: Explicit Guarantees and Clinical Guidelines}

\author{
Gisela Jara1 , Fabiola Werlinger ${ }^{1,2^{*}}$, Rodrigo Cabello ${ }^{1,2}$, Paola Carvajal| ${ }^{1,2}$, Iris Espinoza ${ }^{1,2}$, Rodrigo Giacaman²,3, \\ Ximena Lee ${ }^{1,2}$, Alicia Morales ${ }^{1,2}$, Oscar Arteaga ${ }^{2,4}$, Jorge Gamonal ${ }^{1,2}$.
}

1. Facultad de Odontología, Universidad de Chile, Chile.

2. Centro de Vigilancia y Epidemiología de las

Enfermedades Orales - CEVEO, Chile.

3. Universidad de Talca, Chile.

4. Escuela de Salud Pública. Universidad de Chile, Chile.

* Autor correspondiente. Fabiola Werlinger Cruces. | Sergio Livingstone 943, comuna de Independencia, Santiago, Chile. | Teléfono: (56) 2 29776772. |

E-mail: fwerlinger@odontologia.uchile.cl.

Trabajo recibido el 13/11/2018.

Aprobado para su publicación el 30/11/2018

\section{RESUMEN}

El presente artículo realiza una breve revisión y síntesis sobre las Garantías Explícitas en Salud Bucal vigentes en Chile y las Guías de Práctica Clínica asociadas a su ejercicio. Se muestra una breve cronología de la reforma de salud de la cual se originan, describiendo sus principales pilares de desarrollo. Esta actualización tiene como objetivo orientar a los profesionales odontólogos de los Servicios de Salud, Sociedades Científicas, Universidades y entidades públicas y privadas que desarrollan la práctica odontológica en Chile, en el conocimiento del material existente, validado y disponible a la fecha.

PALABRAS CLAVE:

Salud Bucal; Reforma de Salud; Garantías en Salud; Guías de Práctica Clínica.

Rev. Clin. Periodoncia Implantol. Rehabil. Oral Vol. 11(3); 187-190, 2018.

\section{ABSTRACT}

This article makes a brief review and synthesis of the Explicit Guarantees in Oral Health in force in Chile and the Clinical Practice Guidelines associated with their practice. It shows a brief chronology of the health reform from which they originate, describing their main pillars of development. The purpose of this update is to guide the dental professionals of the Health Services, Scientific Societies, Universities and public and private services that develop the dental practice in Chile, with the knowledge of the existing material, validated and available to date.

KEY WORDS:

Oral Health; Health Care Reform; Insurance Health; Practice Guidelines.

Rev. Clin. Periodoncia Implantol. Rehabil. Oral Vol. 11(3); 187-190, 2018.

\section{REFORMA DE SALUD EN CHILE Y EL ÁMBITO ODONTOLÓGICO}

En Chile durante la década anterior, el sector salud inició una profunda reforma ${ }^{(1,2,3)}$ basada en estrategias y acciones destinadas a satisfacer de mejor manera las necesidades de salud de nuestra población ${ }^{(2,3,4,5)}$.

Esta reforma incluyó una serie de cambios significativos de forma planificada e intencionada en diferentes instancias y funciones del sector $^{(4)}$, con el propósito de aumentar la equidad en el acceso a la salud, la eficiencia en el uso de los recursos y la efectividad de sus actividades $(2,4,5)$

Para su monitoreo se definieron pilares fundamentales y ámbitos de procesos asociados a su operacionalización, conjuntamente a sus resultados intermedios y resultados finales esperados ${ }^{(2,4)}$. En este contexto, y luego de realizado un análisis profundo sobre la situación de salud de la población chilena ${ }^{(2,4,6)}$, se promulga el año 2004 la Ley de Garantías Explícitas en Salud AUGE o Régimen de Garantías Explícitas en Salud GES $(2,4,7)$, base de nuestro nuevo sistema sanitario que debe ser cumplida para todos los chilenos y chilenas, independiente de si pertenecen a Fonasa o Isapres (5,7) $^{(2 .}$

Este régimen de garantías en salud (RGS), fue diseñado para otorgar un conjunto de garantías explícitas y exigibles, que incluyeron: (i) acceso, (ii) calidad, (iii) protección financiera y (iv) oportunidad, para un conjunto priorizado de enfermedades o condiciones de salud ${ }^{(2,3,5)}$ que inicialmente incluyó un listado de 25 problemas prioritarios ${ }^{(5,7,8)}$ los que en la actualidad llegan a $80^{(8,9)}$. La ley otorgaba al Ministerio de Salud la función de seleccionar estos problemas de salud, condiciones o enfermedades, junto con prestaciones garantizadas y las características de acceso y oportunidad(6). Para esto, la misma ley establece que el criterio de priorización se base fundamentalmente en estudios epidemiológicos y económicos ${ }^{(6,10)}$ con la constitución de un consejo consultivo que asesore la toma de estas decisiones ${ }^{(6,11)}$. Los principales criterios de priorización incluyeron estudios de carga de enfermedad y factores de riesgo expresados en "AVISA" $(5,10)$ (suma de los años de vida perdidos, sea por muerte prematura o por incapacidad, atribuibles a cada patología o factor de riesgo)(12); la eficacia y efectividad de las prestaciones que se otorgan de acuerdo a criterios de Medicina Basada en Evidencia; la capacidad real de atención de los sistemas de salud para cada uno de los problemas o condiciones propuestas y las "preferencias sociales" existentes de ese momento ${ }^{(5,7,10)}$. Para el monitoreo y seguimiento de la instalación y funcionamiento de este RGS, se estableció como elemento central la verificación del cumplimiento de la garantía en cada hito de salud que se considerara crítico, fuera ésta la sospecha, confirmación, etapificación o tratamiento del problema ${ }^{(11)}$.

Específicamente en el ámbito odontológico, se realiza entre los años 2003 y 2004 un proceso similar culminando el año 2005 con la priorización de dos problemas de salud: el problema $n^{\circ} 13$ "Fisura labio palatina" $(9,13)$ y el problema $n^{\circ} 23$ "Salud oral integral para niñas y niños de 6 años"(9,14). Este continúa, incorporando el año 2007 el GES de "Urgencias odontológicas ambulatoria"15 y el GES "Atención odontológica integral adulto de 60 años"(16), concluyendo el año 2010 con el GES de "Salud 


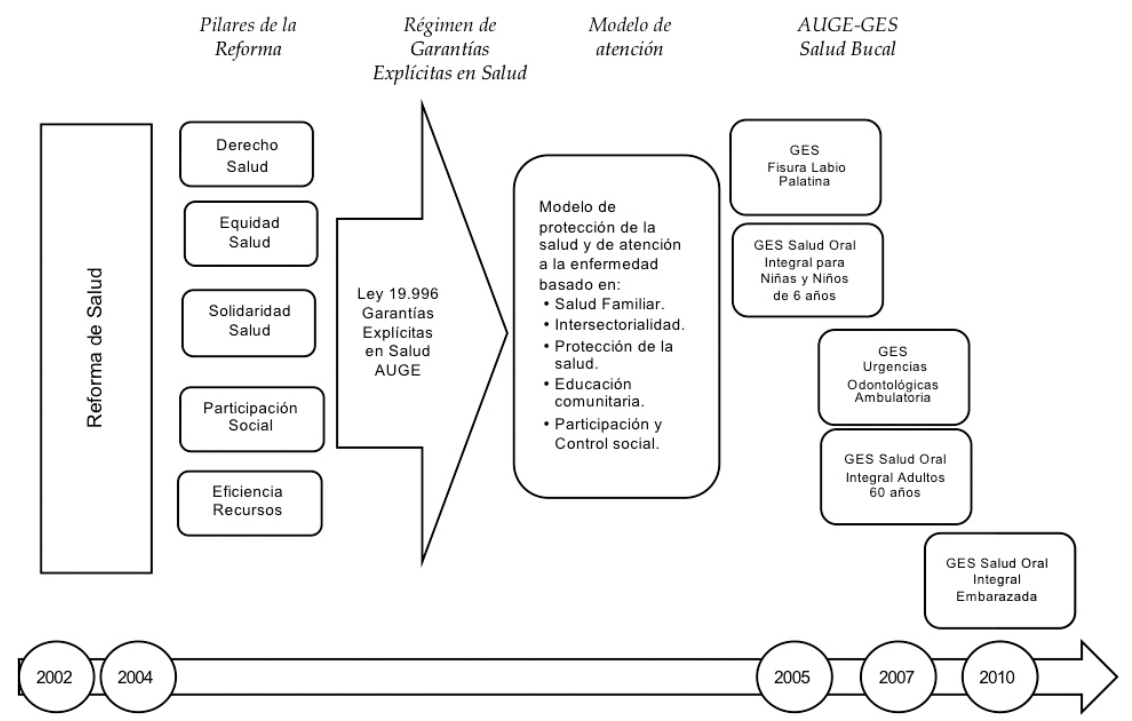

Fuente: elaboración propia.

Figura 1. Contexto histórico de la reforma de salud y el ámbito odontológico en Chile

oral integral de la embarazada"(17), contando a la fecha con un total de 5 problemas de salud oral priorizados en nuestro sistema sanitario, producto de la reforma ${ }^{(9)}$ (Figura 1).

Cada uno de estos problemas, incorpora en su quehacer Guías de Práctica Clínica ${ }^{(18,19)}$ que resumen la mejor evidencia disponible sobre la efectividad de las intervenciones utilizadas en su manejo, formulando recomendaciones destinadas a optimizar el cuidado de los pacientes en la prevención, diagnóstico, tratamiento, seguimiento y/o rehabilitación del problema ${ }^{(19,20)}$. Actualmente el Ministerio de Salud, ha elaborado un manual metodológico incorporando la metodología $\operatorname{GRADE}^{(21)}$ y diversas capacitaciones para mejorar su calidad ${ }^{(20,22)}$, contando con diversas versiones de estas guías (Tabla 1). El detalle de cada GES odontológico y su guía clínica asociada vigente se detalla a continuación.

Table 1. GES odontológicos y guías clínicas asociadas vigentes en Chile

\section{GARANTÍAS EXPLÍCITAS Y GUÍAS CLÍNICAS VIGENTES}

\section{GES Fisura labio palatina}

Incorporada como problema prioritario a partir del Decreto $n^{\circ} 170$ del año 2004 y publicada en el diario oficial el 28 de enero de $2005^{(23)}$, la fisura labio palatina constituye uno de los primeros problemas priorizados de salud oral en la reforma sanitaria(13).

En este, se reconoce a las fisuras labio palatinas como aquellas deficiencias estructurales congénitas generadas por la falta de coalescencia entre algunos de los procesos faciales embrionarios en formación, con distintos grados de severidad. Se incluyen en esta definición la fisura de labio, fisura labio-palatina y fisura palatina aislada, considerando un total de 33 diagnósticos ${ }^{(13)}$. Como beneficiarios de esta

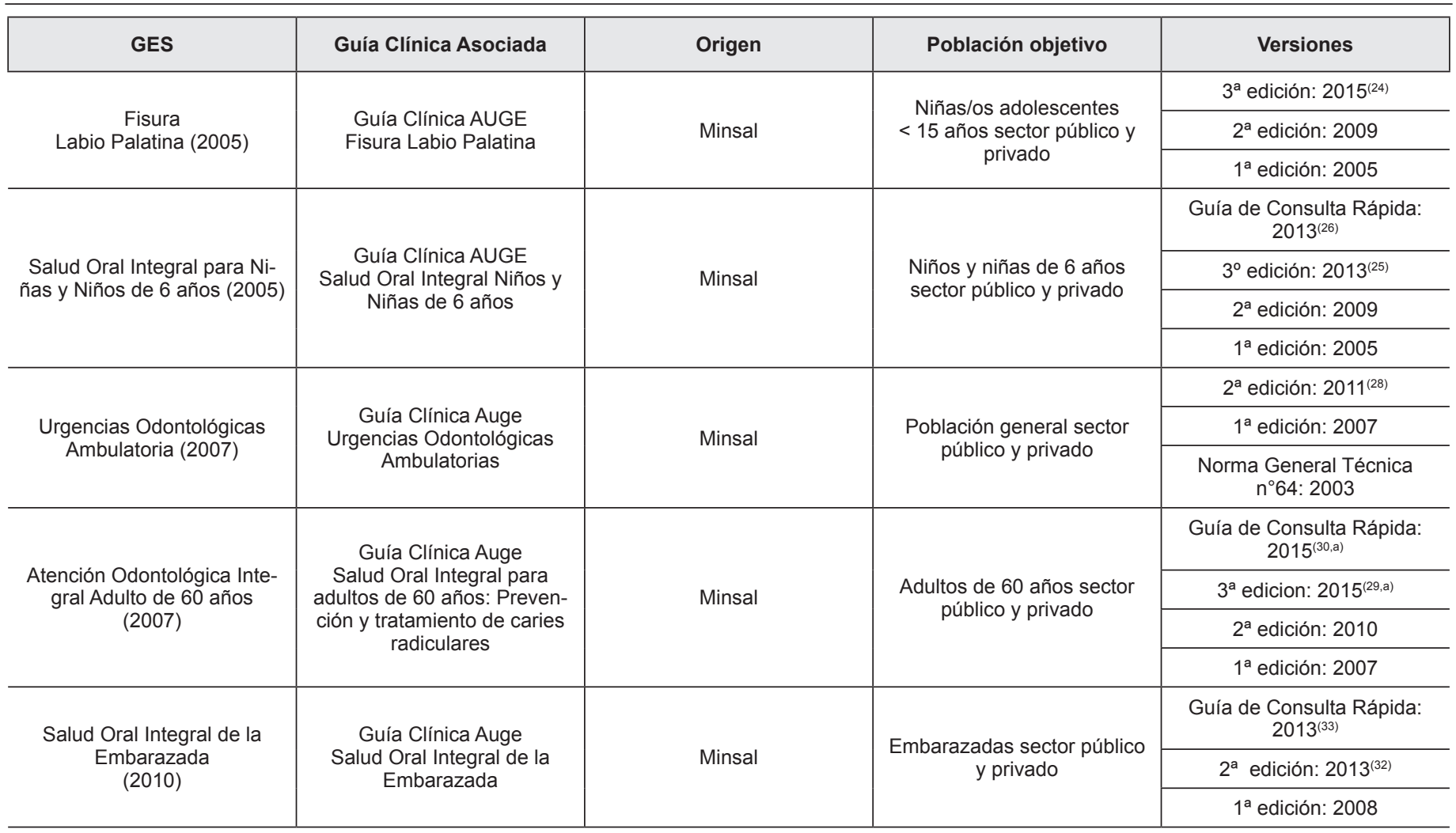

Fuente: elaboración propia. (a)Metodología GRADE. 
garantía, se considera a todos los menores de 15 años con el evento, que hayan nacido a partir del $1^{\circ}$ de julio del 2005 , época en que se promulga la ley que la acoge, otorgando acceso al diagnóstico, tratamiento y rehabilitación ${ }^{(24)}$

\section{Guía clínica vigente:}

"Guía Clínica AUGE Fisura Labio Palatina". 3a edición. Santiago: MINSAL, 2015. Esta guía incluye recomendaciones dirigidas a odontólogos y todos los profesionales que intervienen en la prevención, diagnóstico (prenatal y al nacimiento), tratamiento, seguimiento y rehabilitación de los recién nacidos, niños y adolescentes con fisura de labio, paladar y de labio y paladar. La guía orienta las buenas prácticas de un diagnóstico multidisciplinario, que incluyen acciones desde el periodo de gestación como la suplementación de ácido fólico en la dieta de las madres, y posterior seguimiento, considerando dimensiones de tratamiento clínico-quirúrgico y de salud mental del paciente ${ }^{(21)}$.

\section{GES Salud oral integral para niñas y niños de 6 años}

Incorporado como problema prioritario( ${ }^{(9)}$, y promulgado también como ley el año $2005^{(23)}$, incorpora la definición de salud oral integral como aquella atención odontológica de nivel básico o primario dirigida a educar, prevenir y tratar precozmente al niño de 6 años para mantener en el tiempo una buena salud bucal. La inclusión de este grupo etario se basa en considerar en el ámbito odontológico la edad de 6 años como clave al iniciarse la dentición mixta, etapa en que el diagnóstico oportuno para la conservación de los dientes temporales y la aplicación de medidas de prevención específicas o pesquisa precoz de patologías en dientes definitivos, sumado a la educación, asegurarían la conservación en el tiempo de una buena salud bucal(26). Esta garantía incluye un total de 20 diagnósticos en los beneficiarios que a la fecha de la intervención o tratamiento cuenten con 6 años ${ }^{(14)}$.

\section{Guías clínicas vigentes:}

"Guía Clínica AUGE Salud Oral Integral para Niños y Niñas de 6 años". $3^{a}$ edición. Santiago: MINSAL, 2013. Guía dirigida a odontólogos generales, odontopediatras y personal auxiliar y paramédico del equipo odontológico que estén encargados de proveer la atención integral de nivel primario a los niños de 6 años en establecimiento de salud públicos y privados del país bajo el RGE. La guía aborda recomendaciones para la prevención primaria, basada en intervenciones educativas y el uso de barniz de flúor, conjuntamente al diagnóstico y tratamiento de la caries dental y gingivitis, con técnicas mínimamente invasivas, excluyéndo la atención de urgencias odontológicas ambulatorias (UOA)(25).

"Guía Clínica AUGE 2013. Salud Oral Integral para niños y niñas de 6 años. Guía de consulta rápida”. MINSAL, 2013. Esta guía destaca las recomendaciones para las intervenciones más frecuentes incluidas en el tratamiento odontológico de niños y niñas de 6 años bajo el $\operatorname{RGS}^{(26)}$.

\section{GES Urgencias odontológicas ambulatoria}

Incluido como el problema número $46^{(9,15)}$ a partir del Tercer Decreto $n^{\circ} 44$, se aprueba esta garantía el 9 de enero de $2007^{(27)}$. En él, se definen los eventos de UOA como aquel conjunto de patologías bucomáxilo faciales, de aparición súbita, etiología múltiple y que se manifiestan principalmente con dolor agudo provocando una demanda espontánea de atención. Incluye un total de 8 diagnósticos con tratamiento inmediato para todo beneficiario (adulto o niño) con confirmación diagnóstica dentro de las 24 horas desde la sospecha y entrega de tratamiento inmediato desde su confirmación ${ }^{(15)}$.

\section{Guía clínica vigente:}

"Guía Clínica AUGE Urgencias Odontológicas Ambulatorias". 2a edición. Santiago: MINSAL, 2011. Esta guía está dirigida a aquellos profesionales clínicos que otorgan atención de UOA en población infantil o adulta, sin co-morbilidades, atendidos en centros de salud primaria, servicios de atención primaria de urgencia y servicios de urgencia públicos y privados de nivel secundario y terciario. Estas incluyen patologías como la pericoronaritis, infecciones odontogénicas y el trauma dentoalveolar, entre otras. Sus recomendaciones abordan el manejo clínico de la urgencia, buscando mejorar la eficacia y efectividad de sus tratamientos, estandarizando las prácticas de atención clínica ${ }^{(28)}$.

\section{GES Salud oral integral del adulto de 60 años}

Problema 47 en el listado de problemas prioritarios ${ }^{(9,15)}$, fue incorporado el año $2007^{(27)}$. Consiste en la provisión de atención odontológica de adultos de 60 años realizada por el odontólogo, que va dirigida a educar, prevenir, recuperar y rehabilitar según las necesidades de la salud bucal del paciente. Esto basado en considerarse una población con mayor riesgo de caries por el proceso de envejecimiento, con una alta prevalencia de enfermedades crónicas y enfermedades bucales, uso de medicamentos y vulnerabilidad social ${ }^{(29)}$. Incluye un total de 21 diagnósticos aplicable a todo beneficiario que al consultar cuente con 60 años. El alta de tratamiento ocurre cuando se han completado todos los procedimientos que el paciente requiere. Este tratamiento debe iniciarse dentro de los 90 días de la solicitud de atención ${ }^{(16)}$.

\section{Guías clínicas vigentes:}

"Guía Clínica AUGE Salud Oral Integral para Adultos de 60 Años. MINSAL, 2015. Esta guía va dirigida a los profesionales odontólogos y equipos técnicos en odontología (auxiliares paramédicos, técnicos de nivel superior, entre otros), que atienden a adultos de 60 años o más en centros odontológicos o establecimientos del nivel primario de salud. Las recomendaciones incluyen acciones de prevención específica como el uso de enjuagatorios y pastas fluoruradas a 5000 ppm., hasta el tratamiento restaurador de caries cavitadas ${ }^{(29)}$.

"Guía Consulta Rápida. Salud Oral Integral para adultos de 60 años: Prevención y tratamiento de caries radiculares”. $1^{\text {a }}$ edición. MINSAL, 2015. Actualización que reemplaza las recomendaciones sobre prevención y tratamiento de caries radiculares propuestas en su versión 2010 ${ }^{(30)}$

\section{GES Salud oral integral de la embarazada}

A partir del Decreto 1 promulgado el 6 de enero $2010^{(31)}$, se aprueba la garantía explícita en salud oral de la embarazada correspondiente al problema $\mathrm{n}^{\circ} 66$ del listado de problemas de salud priorizados ${ }^{(9,15)}$ y al último de los problemas del ámbito bucal incorporado a la fecha como garantía explícita.

En este, se define la salud oral integral de la embarazada como una atención odontológica realizada por el odontólogo, según necesidades, dirigida a educar, prevenir, recuperar y rehabilitar la salud de la mujer gestante. Se incluyen en ella 27 diagnósticos a cuyo tratamiento pueden acceder todas las mujeres embarazadas, iniciándose en los 21 días posterior a la solicitud de atención, con una alta integral hasta 15 meses de la primera atención ${ }^{(17)}$.

\section{Guías clínicas vigentes:}

"Guía Clínica AUGE Salud Oral Integral de la Embarazada". Santiago: MINSAL, 2013. Esta guía está dirigida al equipo de salud bucal compuesto por dentistas generales y especialistas, además del personal auxiliar, que entrega atención odontológica a mujeres embarazadas en el sistema público y privado de salud bajo el régimen GES. Se incluyen recomendaciones para la prevención, diagnóstico y tratamiento de caries y enfermedad periodontal en la embarazada, como también recomendaciones para la rehabilitación de piezas perdidas ${ }^{(32)}$.

"Guía Clínica AUGE 2013. Salud Oral Integral de la Embarazada Guía de Consulta Rápida”. MINSAL, 2013. Esta guía complementa las orientaciones técnicas para la educación en salud bucal para embarazadas ${ }^{(33)}$.

\section{CONFLICTO DE INTERÉS}

Ninguno.

\section{FUENTE DE FINANCIAMIENTO}

Sin fuente de financiamiento. 


\section{Bibliografía}

1. Ministerio de Salud de Chile. Hitos de la salud Chilena. Santiago, Chile: Minsal; 2018. [acceso: noviembre 2018]. Disponible en: https://www.minsal.cl/hitos-de-lasalud-chilena/

2. Ministerio de Salud de Chile. Diseño e implementación de una metodología de evaluación, seguimiento y acompañamiento de la Reforma de la Salud de Chile. Chile: Departamento de Desarrollo Estratégico; 2011 [acceso: octubre 2018]. Disponible en: http://www.paho.org/chi/images/PDFs/resumenejecutivo.pdf?ua=1 3. Urriola C, Infante A, Aguilera I, Ormeño H. La reforma de salud Chilena a diez años de su implementación. Sal Pub Mex. 2016;58(5):514-521.

4. Cid C, Salazar E, Tegtmeier R, Riesco X, Muñoz A. Diseño metodológico para medir líneas basales y determinación de línea basal de protección financiera. Chile: Departamento de Estudios y Desarrollo; 2006 [acceso: septiembre 2018]. Disponible en: http://www.supersalud.gob.cl/documentacion/666/articles-3906 recurso 1.pdf 5. Erazo A. La protección social en Chile. El plan AUGE: avances y désafíos. Santiago, Chile: CEPAL; 2011. Serie 238.

6. Valdivieso V, Montero J. El plan AUGE: 2005 al 2009. Rev Med Chile. 2010;138:1040-1046.

7. Régimen de garantías en salud. Ley $\mathrm{N}^{\circ} 19.966$ del 3 de septiembre de 2004. Biblioteca del Congreso Nacional de Chile. [acceso: octubre 2018]. Disponible en: https://www.leychile.cl/Navegar?idNorma=229834

8. Departamento de Estudios, Extensión y Publicaciones. Los cambios en el Régimen de Garantías Explícitas en Salud GES-ex AUGE. Chile: Biblioteca del Congreso Nacional de Chile; 2016 [acceso: agosto 2018]. Disponible en: https://www.bcn.cl/ asesoriasparlamentarias/categorias?id=1\&pag=31\&K=4\&sort=fecha desc

9. Patologías Garantizadas AUGE. Chile: Superintendencia de Salud; 2018 [acceso: octubre 2018]. Disponible en: http://www.supersalud.gob.cl/664/w3propertyname-501.html

10. Castillo C, Aravena M, Freile B, Castillo M, Loayza S, Strappa V. Revisión del proceso de priorización de las Garantías Explicitas en Salud (GES). Informe final. Chile: Ministerio de Salud. Subsecretaría de Salud Pública; 2012.

11. Labbé J, Molina C. Evaluación de los procesos e impactos de la Reforma Sanitaria al Sistema de Salud de Chile. Chile: Ministerio de Salud; 2003 [acceso: septiembre 2018] Disponible en: http://www.supersalud.gob.cl/documentacion/666/ articles-3917 recurso 1 .pdf

12. Ministerio de Salud. Estudio de carga de enfermedad. Informe final. Chile: Subsecretaría de Salud Pública; 2008.

13. Problema de Salud AUGE No13. Fisura labiopalatina. Chile: Superintendencia de Salud; 2018 Material explicativo. [acceso: octubre 2018]. Disponible en: http:// www.supersalud.gob.cl/difusion/665/w3-article-587.html\#accordion_1

14. Problema de Salud Auge $n^{\circ} 23$. Salud oral integral para niñas y niños de 6 años. Chile: Superintendencia de Salud; 2018 Material Explicativo. [acceso: octubre 2018]. Disponible en: http://www.supersalud.gob.cl/difusion/665/w3-article-597.html 15. Problema de Salud Auge $n^{\circ} 46$. Urgencia odontológica Ambulatoria. Material explicativo. Chile: Superintendencia de Salud; 2018 [acceso: octubre 2018]. Disponible en:http://www.supersalud.gob.cl/difusion/665/w3-article-3696.html

16. Problema de Salud Auge $n^{\circ}$ 47. Salud Oral Integral del Adulto de 60 años. Material explicativo. Chile: Superintendencia de Salud; 2018 [acceso: octubre 2018]. Disponible en: http://www.supersalud.gob.cl/difusion/665/w3-article-3697.html

17. Problema de Salud Auge $n^{\circ} 66$. Salud Oral Integral de la Embarazada. Material Explicativo. Chile: Superintendencia de Salud; 2018 [acceso: octubre 2018]. Disponible en: http://www.supersalud.gob.cl/difusion/665/w3-article-5997.html

18. Guías Clínicas Auge. Chile: Ministerio de Salud; 2018 [acceso: agosto 2018] Disponible en: https://diprece.minsal.cl/le-informamos/auge/acceso-guias-clinicas/ guias-clinicas-auge/

19. Guías Clínicas de Salud Bucal. Chile: Ministerio de Salud; 2018 [acceso: agosto 2018]. Disponible en: https://diprece.minsal.cl/programas-de-salud/salud-bucal/ informacion-al-profesional-salud-bucal/guias-clinicas/
20. Ministerio de Salud. Chile. Subsecretaría de Salud Pública. Manual metodológico. Desarrollo de guías de práctica clínica. 2014. [acceso: agosto 2018]. Disponible en: http://www.bibliotecaminsal.cl/wp/wp-content/uploads/2016/04/ Manual-metodologico-GPC-151014.pdf

21. Sanabria A, Rigau D, Rotaeche R, Selva A, Marzo-Castillejo M, Alonso-Coello P. Sistema GRADE: metodología para la realización de recomendaciones para la práctica clínica. Aten Primaria. 2015;47(9):48-55.

22. Pantoja T, Valenzuela L, Léniz J, Castañón C. Guías de práctica clínica en el Régimen de Garantías en Salud: una evaluación crítica de su calidad. Rev Med Chile. 2012;140:1391-1400.

23. Aprueba Garantías Explícitas en Salud del Régimen General de Garantías en Salud. Decreto 170. Biblioteca del Congreso Nacional de Chile. 28 de enero de 2005. [acceso: noviembre 2018]. Disponible en: https://www.leychile.cl/ Navegar?idNorma $=235073$

24. Ministerio de Salud de Chile. Guía clínica AUGE Fisura labiopalatina. $3^{a}$ edición. Santiago, Chile: Subsecretaría de Salud Pública; 2015 [acceso: septiembre 2018]. Disponible en: https://diprece.minsal.cl/wrdprss minsal/wp-content/ uploads/2016/10/guia-FisuraLabioPalatina-2015-CM.pdf

25. Ministerio de Salud. Guía Clínica Auge Salud Oral integral para niños y niñas de 6 años. $3^{a}$ edición. Santiago, Chile: Subsecretaría de Salud Pública; 2013. [acceso: septiembre 2018]. Disponible en: https://diprece.minsal.cl/wrdprss minsal/ wp-content/uploads/2014/12/Salud-Oral-Integral-ni\%C3\%B1os-y-ni\%C3\%B1as-6a\%C3\%B1os.pdf

26. Ministerio de Salud. Guía Clínica Auge. Salud oral integral para niños y niñas de 6 años. Guía consulta rápida. Santiago, Chile: Subsecretaría de Salud Pública; 2013. [acceso: septiembre 2018]. Disponible en: https://diprece.minsal.cl/wrdprss minsal/wp-content/uploads/2014/08/Salud-Oral-Integral-para-ni\%C3\%B1os-yni\%C3\%B1as-de-6-a\%C3\%B1os-Consulta-R\%C3\%A1 pida-2013.pdf

27. Aprueba Garantías Explícitas en Salud del Régimen General de Garantías en Salud. Decreto 44. Biblioteca del Congreso Nacional de Chile. 31 de enero de 2007. [acceso: octubre 2018]. Disponible en: https://www.leychile.cl/Navegar?idNorma=2 $58003 \&$ buscar $=19966$

28. Ministerio de Salud de Chile. Guía de urgencias odontológicas ambulatoria. $2^{2}$ edición. Santiago, Chile: Subsecretaría de Salud Pública; 2011. [acceso: septiembre 2018]. Disponible en: https://www.minsal.cl/portal/url/item/7222b6448161ecb1e040 01011f013f94.pdf

29. Ministerio de Salud. Guía de práctica clínica. Salud oral integral para adultos de 60 Años: Prevención y tratamiento de caries radiculares. Santiago: Minsal, 2015. [acceso: agosto 2018]. Disponible en: https://diprece.minsal.cl/wrdprss minsal/ wp-content/uploads/2016/03/Salud-Oral-Integral-para-adultos-de-60-a\%C3\%B1osPrevenci\%C3\%B3n-y-tratamiento-de-caries-radiculares-2015.pdf

30. Ministerio de Salud. Guía consulta rápida. Salud oral integral para adultos de 60 años: Prevención y tratamiento de caries radiculares. Santiago: Minsal, 2015. [acceso: agosto 2018]. Disponible en: https://diprece.minsal.cl/wrdprss_minsal/wpcontent/uploads/2016/03/Gu\%C3\%ADa-Cl\%C3\%ADnica-Salud-oral-integral-60.pdf 31. Aprueba Garantías Explícitas en Salud del Régimen General de Garantías en Salud. Decreto 1. Biblioteca del Congreso Nacional de Chile. 27 de febrero de 2010. [acceso: octubre 2018]. Disponible en: https://www.leychile.cl/ Navegar?idNorma $=1011549$

32. Ministerio de Salud. Guía clínica Auge salud oral integral de la embarazada. Santiago: MINSAL, 2013. [acceso: septiembre 2018]. Disponible en: https:// diprece.minsal.cl/wrdprss minsal/wp-content/uploads/2014/12/Atenci\%C3\%B3nOdontol\%C3\%B3gica-Integral-Embarazada.pdf

33. Ministerio de Salud. Guía clínica Auge 2013. Salud oral integral de la embarazada. Guía consulta rápida. Santiago: Minsal, 2015 [acceso: septiembre 2018]. Disponible en: https://diprece.minsal.cl/wrdprss minsal/wp-content/uploads/2016/03/Consultarapida.-Salud-oral-embarazada-2013.pdf 